\title{
Pyogenic Granuloma of Buccal Mucosa: An Unusual Presentation
}

${ }^{1}$ Usha Jambunath, ${ }^{2} \mathrm{P}$ Balaji, ${ }^{3} \mathrm{~S}$ Latha, ${ }^{4} \mathrm{MB}$ Sowbhagya

\begin{abstract}
Pyogenic granuloma (PG) or granuloma pyogenicum is a common tumor-like growth of the oral cavity or skin is a kind of inflammatory hyperplasia. It is non-neoplastic in nature and represents an exuberant tissue response to local irritation or trauma. In oral cavity, PG shows striking predilection for gingiva with interdental papilla being most common site in $70 \%$ of cases. Other studies reveal $87.09 \%$ of PG occur on gingiva, $9.67 \%$ occur on lip site and $3.22 \%$ occur with buccal mucosa.

Here is a report of unusual case of extragingival PG occurring on the buccal mucosa.
\end{abstract}

Keywords: Buccal mucosa, Extragingival, Pyogenic granuloma.

How to cite this article: Jambunath $U$, Balaji $P$, Latha $S$, Sowbhagya MB. Pyogenic Granuloma of Buccal Mucosa: An Unusual Presentation. J Health Sci Res 2015;6(1):8-10.

Source of support: Nil

Conflict of interest: None

\section{INTRODUCTION}

Pyogenic granuloma (PG) is a distinctive clinical entity originating as a response of the tissues to a nonspecific infection. It is a tumor-like growth that is considered as an exaggerated, conditioned response to minor trauma. ${ }^{1}$ The name PG is a misnomer since the condition is not associated with pus and does not represent a granuloma histologically. Some authors use the term lobular capillary hemangioma for this lesion. ${ }^{2}$

Hullihens description in 1844 was most likely the first case of PG reported in English literature, but the term, PG or granuloma pyogenicum was introduced by Hartzell in 1904. There are two kinds of PG namely lobular capillary hemangioma and non-lobular capillary hemangioma type which differ in their histological

${ }^{1}$ Postgraduate Student, ${ }^{2}$ Professor and Head, ${ }^{3}$ Professor ${ }^{4}$ Reader

${ }^{1-4}$ Department of Oral Medicine and Radiology, Rajarajeswari Dental College and Hospital, Bengaluru, Karnataka, India

Corresponding Author: Usha Jambunath, Postgraduate Student, Department of Oral Medicine and Radiology Rajarajeswari Dental College and Hospital, Bengaluru, Karnataka, India, Phone: 080-26602712, e-mail: ushadentist@gmail.com features. ${ }^{3}$ Pyogenic granuloma is also known as pregnancy granuloma or pregnancy tumor when occurring in pregnant women, or as vascular epulis, benign vascular tumor and hemangiomatous granuloma. ${ }^{4}$

Clinically, these lesions usually present as single nodule or sessile papule with smooth or lobulated surface reddish pink in color and bleeds on palpation. They may vary in size from a few millimeters to several centimeters. As lesions mature, the vascularity decreases and clinically appears pale pink with firm consistency. The peak prevalence is in teenagers and young adults, with a female predilection of $2: 1$. $^{2}$ Incidence of these lesions increase during pregnancy which may be related to the increased levels of estrogen and progesterone.

\section{CASE REPORT}

A 45 years old female patient reported to the Department of Oral Medicine and Radiology, Rajarajeswari Dental College and Hospital, Bengaluru, with the chief complaint of swelling and pain in the right upper back teeth region, since a week. Patient's history revealed the presence of the growth since 5 years which was initially small in size then gradually increased to attain the present size and experienced pain with the same since 1 week. Pain was dull aching type and intermittent in nature, aggravated while chewing. No history of blood or pus discharge. Patient's medical and family history was non-contributory. Personal history revealed habit of chewing betel leaf and quid twice daily from past 3 years. On general physical examination, patient had normal gait, moderately built and nourished, did not show any signs of systemic disease. All vital signs were within normal limits. Extraoral examination (Fig. 1) revealed no abnormality. On intraoral examination (Fig. 2), a solitary sessile growth measuring approximately $1 \times 1.2 \mathrm{~cm}$, roughly oval in shape was seen on the right buccal mucosa at the line of occlusion in relation to maxillary 3rd molar region. It was pale pink in color with an erythematous area in the center of the surface and also the growth on its surface shows indentations of the tooth. On palpation, all the inspectory findings were confirmed. The swelling was tender and firm in consistency. Other findings include dental caries with respect to 48 , and generalized supragingival calculus with extrinsic stains. 


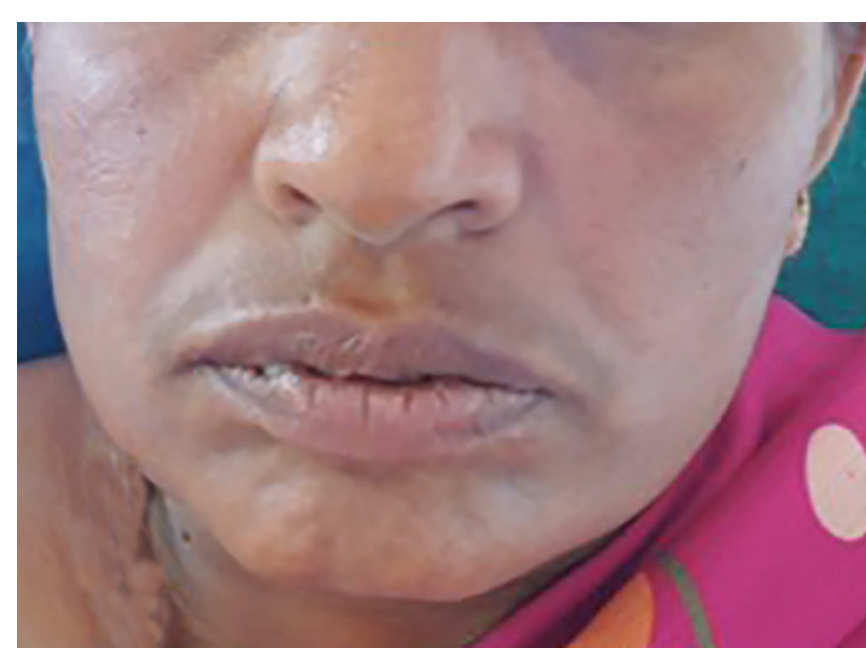

Fig. 1: Extraoral photograph

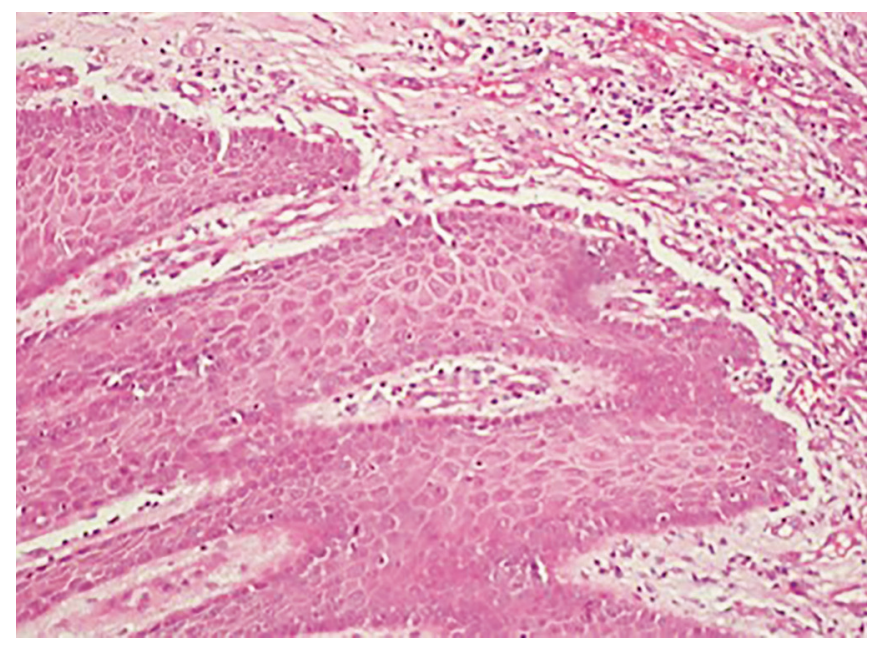

Fig. 3: Photomicrograph histological picture showing connective tissue stroma with numerous engaged blood vessels and budding endothelial cells $(4 x)$

On the basis of history and clinical findings, provisional diagnosis of traumatic/irritational fibroma was considered as the swelling was in the line of occlusion, might be initiated due to cheek bite and has aggravated by continuous trauma from buccal cusps of maxillary 3rd molar. Differential diagnosis of fibrosed PG was considered. Patient was subjected to routine hematological investigations and the results were within normal limits. Excisional biopsy of the growth was done and the sample was subjected for histopathological examination (Fig. 3). Microscopic picture revealed a parakeratotic stratified squamous epithelium which was edematous in the superficial layer and the underlying connective tissue stroma showed numerous engorged blood vessels, budding endothelial cells, chronic inflammatory cell infiltrate with a few areas of mature dense collagen fibers. These findings were suggestive of PG.

Based on clinical and histopathological findings, a final diagnosis of PG of right buccal mucosa was given.

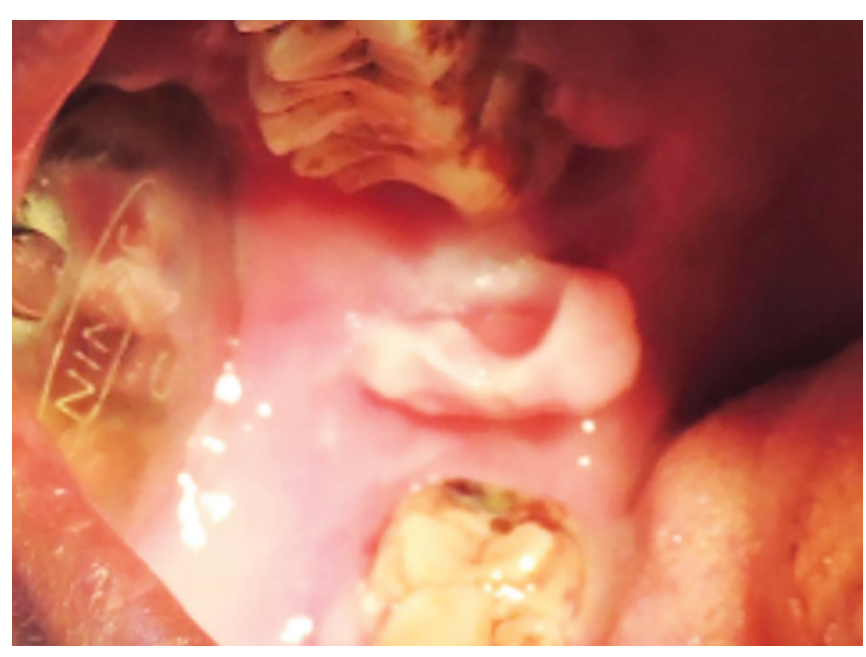

Fig. 2: Swelling on left buccal mucosa

\section{DISCUSSION}

Pyogenic granuloma is the most common of all the oral tumor-like growths. While the terminology implies a benign neoplasm, most if not all fibromas represent reactive focal fibrous hyperplasias due to trauma or local irritation. $^{5}$

Several etiologic factors, such as trauma, injury to a primary tooth, chronic irritation, hormones, drugs, gingival inflammation, pre-existing vascular lesions, chronic irritation due to exfoliation of primary teeth, eruption of permanent teeth, defective fillings in the region of tumor, food impaction, periodontitis, tooth brush trauma, etc. have been suggested.

In previous studies, it has been suggested that trauma can cause release of various endogenous substances including angiogenic factors from the tumor cells and it may also cause disturbances in the vascular system of the affected area. ${ }^{6}$ Because of these changes, the underlying fibrovascular connective tissue becomes hyperplastic and there is proliferation of granulation tissue which leads to the formation of pyogenic granuloma. ${ }^{7}$

Pyogenic granuloma shows striking predilection for gingiva and interdental papilla in about $70 \%$ of cases. Other studies reveal $87.09 \%$ of PG occur on gingiva, and $3.22 \%$ occur on the buccal mucosa. Extragingival PGs tend to occur as a result of trauma. Buccal mucosa, alveolar mucosa of edentulous ridge, palate and lower lip are the sites of extragingival PGs which are very rare. ${ }^{8}$ In the present case, constant trauma either from teeth or occlusion could have been the etiology for the growth on the buccal mucosa.

Clinically, PG presents as a smooth or lobulated mass that is usually pedunculated, although some lesions are sessile. The surface is characteristically ulcerated and ranges from pink to red to purple, depending on the age of the lesion. Initially, PGs are highly vascular in appearance; 
older lesions tend to become more collagenized and pale pink. They vary from small growth of only a few millimeters in size to larger lesions that may measure up to several centimeters in diameter. Typically, the mass often bleeds easily because of its extreme vascularity. Pyogenic granulomas may exhibit rapid growth, which may be alarming to both the patient and the clinician, who may fear that the lesion might be of vascular origin or malignant. ${ }^{9}$

Pyogenic granuloma may occur at all ages but is predominantly seen in the second decade of life in young adult females, possibly because of the vascular effects of female hormones. Clinical diagnosis of extragingival PG can be a challenging task. Such an atypical presentation leads to erroneous diagnosis of other serious lesion, such as peripheral giant cell granuloma, peripheral odontogenic or ossifying fibromas, vascular lesions, such as hemangiomas, and malignant lesions, such as Kaposi's sarcoma, squamous cell carcinoma, basal metastatic carcinoma, etc. which have similar clinical appearance. ${ }^{8}$

Although PG can be diagnosed clinically with considerable accuracy, radiographic and histopathological investigations, aid in confirming the diagnosis and treatment. Radiographs are advised to rule out bony destruction suggestive of malignancy or to identify a foreign body. ${ }^{2}$

Microscopic features show a highly vascular proliferation that resembles granulation tissue. Numerous small and larger endothelium-lined channels are formed that are engorged with red blood cells. These vessels some times are organized in lobular aggregates and some pathologists require this lobular arrangement for the diagnosis (lobular capillary hemangioma). The surface is usually ulcerated and replaced by a thick fibrinopurulent membrane. A mixed inflammatory cell infiltrate of neutrophils, plasma cells, and lymphocytes is evident. Neutrophils are most prevalent near the ulcerated surface. Chronic inflammatory cells are found deeper in the specimen. Older lesions may have areas with a more fibrous appearance., ${ }^{9,10}$

The treatment of choice is conservative surgical excision. For gingival lesions, excising the lesion down to the periosteum and scaling adjacent teeth to remove any calculus and plaque that may be a source of continuing irritation is recommended. Pyogenic granuloma occasionally recurs, and a re-excision is necessary. The prognosis is excellent, and the lesion usually does not recur unless inadequately removed. ${ }^{11}$

\section{CONCLUSION}

Pyogenic granuloma is a non-neoplastic growth in the oral cavity and usually do not attain an unusually large size. Considering these characteristics, PG can be adequately treated with correct diagnosis and proper treatment. Fifteen percent recurrence rate has been reported, however, recurrences after surgery of extragingival PG is uncommon.

\section{REFERENCES}

1. Rajendran R, Sivapathasundaram B. Shafer's Textbook of Oral Pathology. 6th ed. Elsevier Delhi; 2009. p. 328-330.

2. Amirchaghmaghi M, Falaki F, Mohtasham N, Mozafari PM. Extragingival pyogenic granuloma: a case report. Cases J 2008;1(1):371-374.

3. Rizwanulla T, et al. Pyogenic granuloma: a case report. Health Renaissance 2010;8(3):196-198.

4. Gonclas ES, et al. Pyogenic granuloma on the upper lip: an unusual location. J Appl Oral Sci 2010;18(5):538-541.

5. Verma, et al. Pyogenic granuloma-hyperplastic lesion of the gingiva: a case reports. Open Dent J 2012;6:153-156.

6. Kamal R, Dahiya P, Puri A. Oral pyogenic granuloma: various concepts of etiopathogenesis. J Oral Maxillofac Pathol 2012;16(1):79-82.

7. Saikhedkar R, Shrivastava S, Melkundi M, Viswanathan V. Pyogenic granuloma-a case report. Int J Dent Clin 2011;3(3): 87-88.

8. Sulabha AN, Choudhari S, Suchitra G. Pyogenic granulomareport of three cases in rare sites. Bang J Med Sci 2013;12(4): 445-449.

9. Neville BW, Damm DD, Allen CM, Bouquot JE. Oral and Maxillofacial Pathology. 3rd ed. Elsevier Philadelphia; 2009. p. 517-519.

10. Regezi JA, Sciubba JJ, Jordan RC. Oral Pathology: Clinical Pathologic Considerations. 4th ed. Philadelphia, WB Saunders; 2003. p. 115-116.

11. Venugopal S, Shobha KS, Netravathi TD. Pyogenic granuloma: a case report. J Dent Sci Res 2013;1(1):80-85. 